\title{
List of Theoretical Physics Papers for Which Their Authors were Awarded with the Nobel Prize in Physics
}

Rainer W. Kühne

Bürgerstr. 4, 38118 Braunschweig, Germany

kuehne70@gmx.de

I present a list of 66 theoretical physics papers for which their authors were awarded with the Nobel Prize in Physics. These include Lorentz 1902 [1], von Laue 1914 [2], Planck 1918 [3, 4], Einstein 1921 [5], Bohr 1922 [6], de Broglie 1929 [7], Heisenberg 1932 [8], Schrödinger 1933 [9], Dirac 1933 [10], Pauli 1945 [11], Yukawa 1949 [12], Born 1954 [13, 14], Lee and Yang 1957 [15], Tamm and Frank 1958 [16], Landau 1962 [17], Wigner 1963 [18, 19], Goeppert-Mayer 1963 [20], Jensen 1963 [21], Tomonaga 1965 [22], Schwinger 1965 [23-26], Feynman 1965 [27, 28], Bethe 1967 [29, 30], Gell-Mann 1969 [31], Alfvén 1970 [32], Néel 1970 [33], Bardeen, Cooper and Schrieffer 1972 [34, 35], Josephson 1973 [36], Bohr and Mottelson 1975 [37], Anderson 1977 [38], Glashow 1979 [39], Weinberg 1979 [40], Salam 1979 [41], Wilson 1982 [42, 43], Chandrasekhar 1983 [44], Fowler 1983 [45], Laughlin 1998 [46], 't Hooft and Veltman 1999 [47-50], Ginzburg 2003 [51, 52], Abrikosov 2003 [53, 54], Leggett 2003 [55], Gross and Wilczek 2004 [56], Politzer 2004 [57], Glauber 2005 [58], Nambu 2008 [59], Kobayashi and Maskawa 2008 [60], Englert 2013 [61], Higgs 2013 [62, 63], Kosterlitz and Thouless 2016 [64], Haldane 2016 [65, 66].

[1] H. A. Lorentz, "La théorie electromagnétique de Maxwell et son application aux corps mouvants", Archives néerlandaises des Sciences exactes et naturelles 25 (1892) 363-552.

[2] W. Friedrich, P. Knipping, M. von Laue, "Interferenz-Erscheinungen bei Röntgenstrahlen", Sitzungsberichte der mathematisch-physikalischen Klasse der Königlich-Bayerischen Akademie der Wissenschaften zu München (1912) 303-322.

[3] M. Planck, "Über eine Verbesserung der Wienschen Spectralgleichung", Verhandlungen der deutschen physikalischen Gesellschaft 2 (1900) 202-204.

[4] M. Planck, "Zur Theorie des Gesetzes der Energieverteilung im Normalspectrum", Verhandlungen der deutschen physikalischen Gesellschaft 2 (1900) 237-245.

[5] A. Einstein, "Über einen die Erzeugung und Verwandlung des Lichtes betreffenden heuristischen Gesichtspunkt", Annalen der Physik 17 (1905) 132-148.

[6] N. Bohr, "On the Constitution of Atoms and Molecules", Philosophical Magazine 26 (1913) 1-25.

[7] L. de Broglie, "Recherches sur la théorie des quanta", Doctoral thesis (1924).

[8] W. Heisenberg, "Über quantentheoretische Umdeutung kinematischer und mechanischer Beziehungen", Zeitschrift für Physik 33 (1925) 879-893.

[9] E. Schrödinger, "Quantisierung als Eigenwertproblem", Annalen der Physik 79 (1926) 361-376.

[10] P. A. M. Dirac, "The quantum theory of the electron", Proceedings of the Royal Society of London A: Mathematical, Physical and Engineering Sciences 117 (1928) 610-624. 
[11] W. Pauli, "Über den Zusammenhang des Abschlusses der Elektronengruppen im Atom mit der Komplexstruktur der Spektren", Zeitschrift für Physik 31 (1925) 765-783.

[12] H. Yukawa, "The prediction and discovery of pions and muons", Proceedings of the Physico-Mathematical Society of Japan 17 (1935) 48-57.

[13] M. Born, "Zur Quantenmechanik der Stoßvorgänge", Zeitschrift für Physik 37 (1926) 863-867.

[14] M. Born, "Quantenmechanik der Stoßvorgänge", Zeitschrift für Physik 38 (1926) 803827.

[15] T. D. Lee, C. N. Yang, "Question of parity conservation in weak interactions", Physical Review 104 (1956) 254-258.

[16] I. E. Tamm, I. M. Frank, Doklady Akademii nauk SSSR 14 (1937) 107-112.

[17] L. Landau, "Theory of the Superfluidity of Helium II", Physical Review 60 (1941) 356358.

[18] E. Wigner, "Einige Folgerungen aus der Schrödingerschen Theorie für die Termstrukturen", Zeitschrift für Physik 43 (1927) 624-652.

[19] E. Wigner, "Über die Operation der Zeitumkehr in der Quantenmechanik", Nachrichten von der Gesellschaft der Wissenschaften zu Göttingen, Mathematisch-Physikalische Klasse (1932) 546-559.

[20] M. Goeppert-Mayer, "On closed shells in nuclei", Physical Review 74 (1948) 235-239.

[21] O. Haxel, J. H. D. Jensen, H. E. Suess, "On the 'magic numbers' in nuclear structure", Physical Review 75 (1949) 1766-1766.

[22] S. Tomonaga, "On a Relativistically Invariant Formulation of the Quantum Theory of Wave Fields", Progress of Theoretical Physics 1 (1946) 27-42.

[23] J. Schwinger, "On quantum-electrodynamics and the magnetic moment of the electron", Physical Review 73 (1948) 416-417.

[24] J. Schwinger, "Quantum electrodynamics. I. A covariant formulation", Physical Review 74 (1948) 1439-1461.

[25] J. Schwinger, "Quantum electrodynamics. II. Vacuum polarization and self-energy", Physical Review 75 (1949) 651-679.

[26] J. Schwinger, "Quantum electrodynamics. III. The electromagnetic properties of the electron—radiative corrections to scattering", Physical Review 76 (1949) 790-817.

[27] R. P. Feynman, "The theory of positrons", Physical Review 76 (1949) 749-759.

[28] R. P. Feynman, "Space-time approach to quantum electrodynamics", Physical Review 76 (1949) 769-789. 
[29] H. A. Bethe, "Energy production in stars", Physical Review 55 (1939) 103-103.

[30] H. A. Bethe, "Energy production in stars", Physical Review 55 (1939) 434-456.

[31] M. Gell-Mann, "The Eightfold Way: A Theory of strong interaction symmetry", in: M. Gell-Mann, Y. Ne'eman, "The Eightfold Way (Benjamin Press, 1964) 11-57.

[32] H. Alfvén, "Existence of electromagnetic-hydrodynamic waves", Nature 150 (1942) 405-406.

[33] L. Néel, "Proprietes magnetiques des ferrites-ferrimagnetisms et antiferromagnetisme", Annales de Physique 3 (1948) 137-198.

[34] J. Bardeen, L. N. Cooper, J. R. Schrieffer, "Microscopic theory of superconductivity", Physical Review 106 (1957) 162-164.

[35] J. Bardeen, L. N. Cooper, J. R. Schrieffer, "Theory of superconductivity", Physical Review 108 (1957) 1175-1204.

[36] B. D. Josephson, "Possible new effects in superconductive tunneling", Physics Letters 1 (1962) 251-253.

[37] A. Bohr, B. R. Mottelson, "Collective and individual-particle aspects of nuclear structure", Det Kongelige Danske Videnskabernes Selskab - Matematisk-fysiske Meddelelser 27 n. 16 (1953) 1-174.

[38] P. W. Anderson, "Absence of diffusion in certain random lattices", Physical Review 109 (1958) 1492-1505.

[39] S. L. Glashow, "Partial-symmetries of weak interactions", Nuclear Physics 22 (1961) 579-588.

[40] S. Weinberg, "A model of leptons", Physical Review Letters 19 (1967) 1264-1266.

[41] A. Salam, "Weak and electromagnetic interactions", in: N. Svartholm, "Elementary Particle Physics" (Almqvist and Wiksell, 1968) 367-377.

[42] K. G. Wilson, "Renormalization group and critical phenomena. I. Renormalization group and the Kadanoff scaling picture", Physical Review B 4 (1971) 3174-3183.

[43] K. G. Wilson, "Renormalization group and critical phenomena. II. Phase-space cell analysis of critical behavior", Physical Review B 4 (1971) 3184-3205.

[44] S. Chandrasekhar, "The maximum mass of ideal white dwarfs", Astrophysical Journal 74 (1931) 81-82.

[45] E. M. Burbidge, G. R. Burbidge, W. A. Fowler, F. Hoyle, "Synthesis of the elements in stars", Reviews of Modern Physics 29 (1957) 547-647.

[46] R. B. Laughlin, "Anomalous quantum Hall effect: an incompressible quantum fluid with fractionally charged excitations", Physical Review Letters 50 (1983) 1395-1398. 
[47] G. 't Hooft, "Renormalization of massless Yang-Mills fields", Nuclear Physics B 33 (1971) 173-199.

[48] G. 't Hooft, "Renormalizable lagrangians for massive Yang-Mills fields", Nuclear Physics B 35 (1971) 167-188.

[49] G. 't Hooft, M. Veltman, "Regularization and renormalization of gauge fields", Nuclear Physics B 44 (1972) 189-213.

[50] G. 't Hooft, M. Veltman, "Combinatorics of gauge fields", Nuclear Physics B 50 (1972) 318-353.

[51] L. D. Landau, V. L. Ginzburg, Zhurnal Eksperimentalnoi i Teoreticheskoi Fiziki 20 (1950) 546-568.

[52] L. D. Landau, V. L. Ginzburg, "On the Theory of Superconductivity", Soviet Physics Journal of Experimental and Theoretical Physics 20 (1950) 1064-1082.

[53] A. A. Abrikosov, Zhurnal Eksperimentalnoi i Teoreticheskoi Fiziki 32 (1957) 1442-1452.

[54] A. A. Abrikosov, "On the Magnetic Properties of Superconductors of the Second Group", Soviet Physics - Journal of Experimental and Theoretical Physics 5 (1957) 11741182.

[55] A. J. Leggett, "Interpretation of Recent Results on He 3 below 3 mK: A New Liquid Phase?", Physical Review Letters 29 (1972) 1227-1230.

[56] D. J. Gross, F. Wilczek, "Ultraviolet behavior of non-abelian gauge theories", Physical Review Letters 30 (1973) 1343-1346.

[57] H. D. Politzer, "Reliable perturbative results for strong interactions?", Physical Review Letters 30 (1973) 1346-1349.

[58] R. J. Glauber, "Photon correlations", Physical Review Letters 10 (1963) 84-86.

[59] Y. Nambu, "Axial vector current conservation in weak interactions" , Physical Review Letters 4 (1960) 380-382.

[60] M. Kobayashi, T. Maskawa, "CP-violation in the renormalizable theory of weak interaction", Progress of theoretical Physics 49 (1973) 652-657.

[61] F. Englert, R. Brout, "Broken symmetry and the mass of gauge vector mesons", Physical Review Letters 13 (1964) 321-323.

[62] P. W. Higgs, "Broken symmetries, massless particles and gauge fields", Physics Letters 12 (1964) 132-133.

[63] P. W. Higgs, "Broken symmetries and the masses of gauge bosons", Physical Review Letters 13 (1964) 508-509.

[64] J. M. Kosterlitz, D. J. Thouless, "Ordering, metastability and phase transitions in twodimensional systems", Journal of Physics C: Solid State Physics 6 (1973) 1181-1203. 
[65] F. D. M. Haldane, "Continuum dynamics of the 1-D Heisenberg antiferromagnet: identification with the O (3) nonlinear sigma model", Physics Letters A 93 (1983) 464-468.

[66] F. D. M. Haldane, "Nonlinear field theory of large-spin Heisenberg antiferromagnets: semiclassically quantized solitons of the one-dimensional easy-axis Néel state", Physical Review Letters 50 (1983) 1153-1156. 